\title{
Tryptophan Metabolism and Cancer Progression
}

\author{
Kenneth K. Wu*
}

Institute of Cellular and System Medicine, National Health Research Institutes, Zhunan, Taiwan and Institute of Biotechnology, National Tsing-Hua University, Hsinchu, Taiwan

\begin{abstract}
Intracellular tryptophan (Trp) is catabolized to a large repertoire of metabolites via two major pathways: indoleamine and tryptophan 2, 3-dioxygenases (IDO/TDO) and Trp hydroxylase (TPH) pathways. The catabolites possess diverse biological activities and carry out various physiological functions. Several catabolites such as kynurenine (Kyn) and serotonin promote while melatonin and 5-methoxytryptophan (5-MTP) suppress cancer growth and metastasis. Cancer cell-derived Kyn enhances cancer growth and evasion of immunosurveillance by interacting with cancer cell and immune cell membrane aryl hydrocarbon receptors (AHR), respectively. Serotonin exerts its tumorpromoting activities through type 1 and type 2 serotonin receptors. 5-MTP and melatonin suppress cancer growth and metastasis by common mechanisms, i.e., inhibition of p300 histone acetyltransferase (HAT) and NF-kB activation, and suppression of cyclooxygenase-2 and cytokine transcription. Both metabolites block p38 MAPK signaling pathway. Human cancer tissues express increased levels of IDO, TDO and kynurenine monooxygenase (KMO) which are correlated with reduced patient survival. In summary, cancer Trp metabolism regulates cancer growth and metastasis by complex mechanisms. 5-MTP and melatonin provide valuable lead to develop new drugs for chemo-prevention and adjuvant therapy of cancer.
\end{abstract}

Keywords: Tryptophan metabolites, cancer progression, melatonin, 5-methoxytryptophan, kynurenine.

\section{INTRODUCTION}

L-tryptophan (Trp) is an essential amino acid required for protein synthesis. Its intracellular pool serves as a substrate for synthesis of bio-active metabolites with diverse biological activities and physiological functions. Trp metabolites regulate immune responses, inflammation, neurotransmission, circadian rhythm, sleep, cellular senescence and vascular protection in a cell-specific manner. They are produced via two pathways: IDO/TDO and TPH pathways (Figure 1). IDO (indolamine 2, 3 dioxygenase) is expressed in immune cells and catalyzes the synthesis of kynurenine (Kyn) [1,2] (Figure 1A). Two IDO isoforms, i.e., IDO-1 and IDO-2 have been identified. IDO-1 is well characterized and its expression and immune-modulating activities have been extensively investigated while the function of IDO2 is less clear [1-4]. IDO-1 is constitutively expressed in placenta and dendritic cells and induced by interferon- $\gamma$ (IFY) in a large number of tissues [5]. TDO (tryptophan 2, 3-dioxygenase) is genetically and structurally distinct from but catalytically similar to IDO. It is expressed in liver cells which accounts for most of Kyn synthesis under physiological conditions [6]. Kyn exerts immunesuppressive actions through induction of $T$ cell apoptosis and upregulation of regulatory $T$ cells (Treg) [7]. In addition, it regulates skeletal muscle and stem cell functions and contributes to inflammation,

*Address correspondence to this author at the Institute of Cellular and System Medicine, National Health Research Institutes, 35 Keyan Road, Zhunan Town, Miaoli County 35053, Taiwan; Tel: +886-37-246-166 ext. 37501;

Fax: +886-37-587-408; E-mail: kkgo@nhri.org.tw neurodegeneration and tumoriness [7]. Kyn is further catabolized to a series of metabolites such as 3hydroxykynurenine, 3-hydroxyathranilic acid, quinolinic acid and nicotinate ribonucleotide, a precursor of NAD (Figure 1A). These catabolic products perform diverse functions.

The TPH (tryptophan hydroxylase) pathway generates three bio-active metabolites: serotonin, melatonin and 5-methoxytryptophan (5-MTP, also known as cytoguardin) (Figure 1B). There are two TPH isoforms. TPH-2 is expressed in neurons while TPH-1 is expressed in peripheral tissues $[8,9]$. Both isoforms are detected in pineal cells. TPH-1 or -2 catalyzes conversion of L-tryptophan to 5-hydroxytryptophan (5HTP) (Figure 1B). In serotonergic neurons, TPH-2 derived 5-HTP is converted to 5-hydroxytryptamine (5$H T$, serotonin) by aromatic L-amino acid decarboxylase (AADC) [10]. Serotonin is an important neurotransmitter regulating behavior, mood and learning [11]. Only a small fraction of body serotonin is produced in central nervous system. A vast majority ( $95 \%$ ) of serotonin is produced in peripheral tissues mostly in enterochromaffin cells of the gut. Adipocytes, osteoclasts and vascular cells also produce serotonin [12]. Serotonin produced by peripheral tissues carries out diverse functions including regulation of vascular tone and gastrointestinal function [12]. In pineal and retinal cells, serotonin serves as an intermediate metabolite for melatonin synthesis: serotonin is converted to $\mathrm{N}$-acetyl-serotonin (N-acetyl 5hydroxytryptamine) by arylalkylamine $\mathrm{N}$-acetyl transferase (AANAT) [13] (Figure 1B). The final step of 


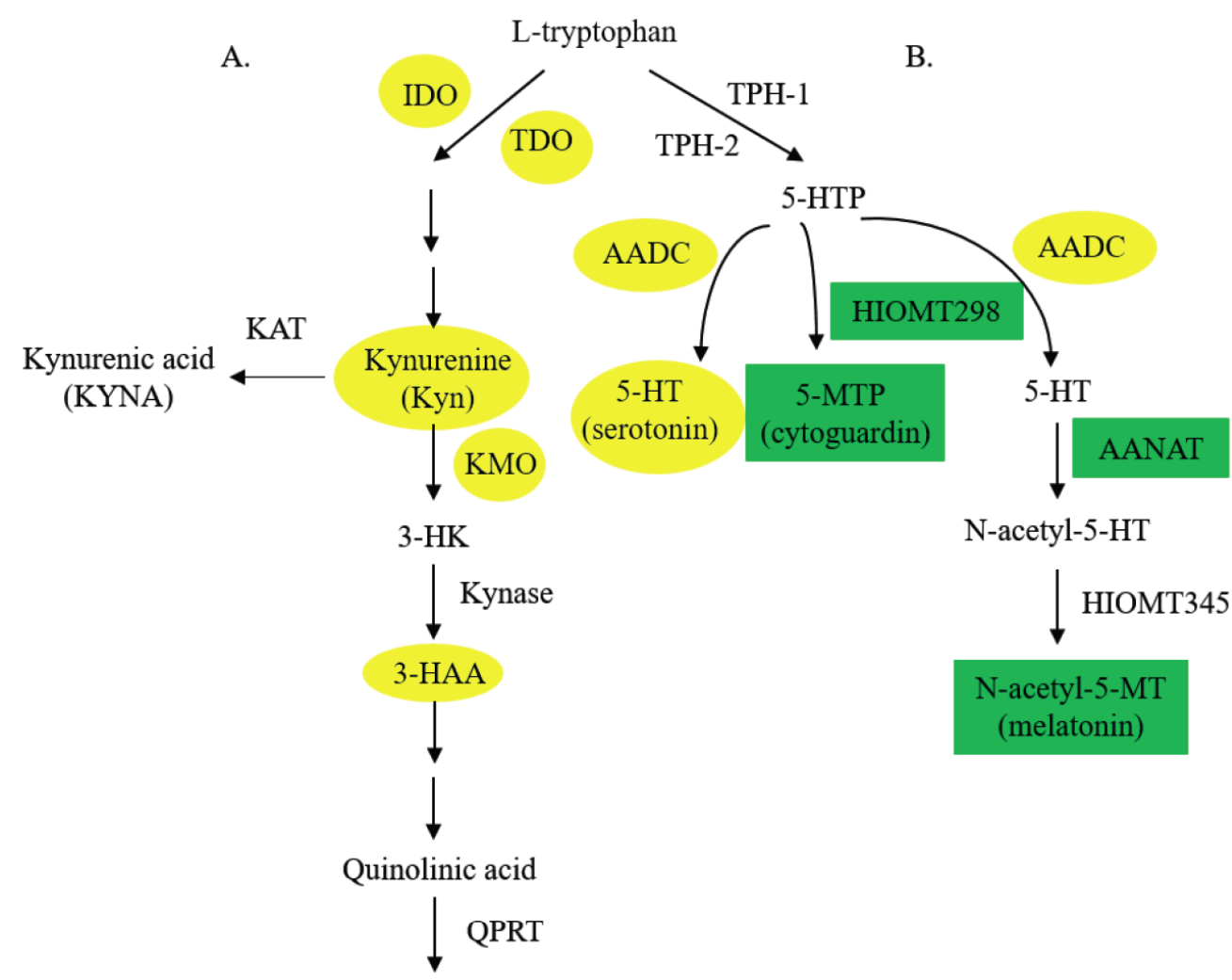

Nicotinate ribonucleotid

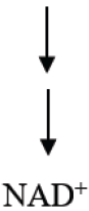

Figure 1: L-tryptophan catabolic pathways. Yellow denotes pro-cancer and green denotes anti-cancer enzymes or metabolites.

melatonin synthesis is catalyzed by $\mathrm{N}$-acetylserotonin O-methyltransferase (ASMT, generally known as hydroxyindole O-methyltransferase, HIOMT). ASMT catalyzes transfer of methyl group from a methyl donor, $\mathrm{S}$-adenosylmethionine (SAM) to $\mathrm{N}$-acetylserotonin to form $\mathrm{N}$-acetyl-5-methoxytryptamine (melatonin) [13] (Figure 1B). Melatonin appears to be selectively produced in pineal and retinal cells. Melatonin synthesis is controlled by circadian rhythm through AANAT which is expressed in dark and suppressed by light [14]. Melatonin plays a physiological role in regulating circadian rhythm and sleep [15]. The third bioactive metabolite in the TPH pathway is 5-MTP which was recently uncovered as an anti-inflammatory and anti-tumor factor [16]. 5-MTP synthesis from 5HTP is also catalyzed by HIOMT. However, a different HIOMT mRNA isoform distinct from the melatonincatalyzing isoform is involved in 5-MTP synthesis [17]. 5-MTP is produced by fibroblasts, mesenchymal stromal cells, vascular endothelial and smooth muscle cells, and epithelial cells [18]. It is secreted into the extracellular milieu via Golgi vesicular transport, where it exerts its biological actions [18].

Extensive investigations have revealed that cancer cells express enzymes to synthesize Kyn and serotonin. Importantly, they utilize these metabolites to promote their own growth. On the other hand, they are the target of melatonin and 5-MTP which suppress their proliferation and migration. Thus, there is an intimate relationship between Trp metabolism and cancer progression. This review will focus on cancer cell Trp metabolism and its response to Trp metabolites. Trp metabolites and catabolic enzymes as potential prognostic biomarkers will be discussed.

\section{CANCER CELLS PRODUCE TUMOR- PROMOTING METABOLITES}

Cancer cells express enzymes such as IDO-1, TDO and kynurenine monooxygenase to produce Kyn and 
its downstream catabolites such as 3hydroxyanthranilic acid (3-HAA). In addition, they express TPH-1 and AADC to produce serotonin. Kyn, 3-HAA and serotonin possess tumor-promoting activities and play an important role in cancer progression.

\subsection{IDO or TDO-Derived Kyn in Cancer Cells Drives Cancer Growth via Aryl Hydrocarbon Receptor (AHR)}

IDO-1 is widely expressed in human cancers [19] while expression of TDO in cancers is selective [20]. Some cancers such as malignant glioma express both enzymes [21]. It is unclear whether expression of IDO1 or TDO has different impacts on cancer progression. Nor is it clear how each enzyme contributes to Kyn production when both enzymes are concurrently expressed. Nevertheless, Kyn has been shown to directly promote cancer cell proliferation and migration via aryl hydrocarbon receptors expressed on cancer cells $[22,23]$. Furthermore, it enhances cancer growth and metastasis by suppressing host immune response to facilitate cancer evasion of immune surveillance [24]. Kyn suppresses the activities of natural killer and $T$ killer cells, inhibits antigen-processing cells and induces regulatory $T$ cell [25-28]. Immunosuppressive effects of Kyn on T-lymphocytes, dendritic cells, monocytes/macrophages and natural killer cells are mediated at least in part through interaction with AHR on the immune cells [29].

AHR, expressed in a wide variety of cancers, is a cytosolic receptor which interacts with exogenous ligands notably environmental toxins such as dioxins and endogenous receptors such as Kyn [21]. Kynbound AHR translocates to nucleus where it forms a heterodimer with ARNT (AHR nuclear translocator). AHR/ARNT complex binds to AHR response elements and promotes expression of genes that enhance cancer cell proliferation and migration [22,29]. Cancer cell-derived Kyn also binds AHR in immune cells thereby altering immune cell activities and inducing phenotypic changes resulting in suppressed immune response and ineffective cancer immune surveillance [29]. Thus, Kyn derived from cancer cell IDO-1 or TDO exerts multiple biological actions by interacting with AHR expressed in cancer cells and immune cells.

\subsection{AADC-Catalyzed Serotonin Produced by Cancer Cells Promotes Cancer Cell Proliferation}

Serotonin is synthesized and secreted from several types of cancer cells including colorectal, breast, hepatocellular and cholangial cancer cells [30-33]. The secreted serotonin acts locally to increase cancer cell proliferation through paracrine mechanisms such as angiogenesis [30]. Serotonin promotes growth of prostate, breast, bladder, colorectal, hepatocellular, cholangial and small cell lung cancer cells by interacting primarily with $5-\mathrm{HT}$ receptors (5-HTR) $1 \mathrm{~A}$, 1B, 2A and 2B [34].

Serotonin is produced from Trp via two enzymatic steps: 1). TPH converts Try to 5-HTP and 2). AADC converts 5-HTP to 5-HT. Genome-scale metabolic network analysis of 2000 human breast cancer tissues has classified active serotonin production as a poor prognostic group [33]. Active serotonin production is attributed to upregulation of AADC. AADC was originally discovered in catecholamine-producing neurons as an enzyme catalyzing conversion to LDOPA to dopamine and hence was named DOPA decarboxylase (DDC) [10]. It was subsequently found that it catalyzes conversion of 5-HTP to 5hydroxytryptamine (5-HT, serotonin). As the enzyme catalyzes decarboxylation of aromatic L-amino acids to generate amines, it is also called aromatic L-amino acid decarboxylase (AADC). AADC is encoded by a single gene with two alternative promoters which drive the expression of two mRNA isoforms through alternative splicing.. The mRNA isoforms are expressed in cell-selective manner: neural isoform and non-neural isoform $[35,36]$. Alternative splicingproduced mRNA isoforms generate two distinct proteins: 480 aa- and 442 aa proteins [37]. The 480 aa isoform is active in catalyzing the formation of serotonin and dopamine. The 442 aa isoform is catalytically inactive and may have a different function [37].

Non-neural mRNA isoform is expressed in kidney and liver as well as in cancer cells. Small cell lung cancer (SCLC) cells which are of neuroendocrine origin express neural isoform [38]. AADC is abundantly expressed in human SCLC tissues and is associated with a better prognosis [39]. In a study with human colorectal cancer, AADC was detected in about $25 \%$ of tissues which was associated with early cancer stage and longer survival [40]. It is to be noted that the sample size in this study is small and may not be sufficient for drawing conclusion. By contrast, AADC expression levels in prostate cancer tissue were reported to be associated with a poor prognosis with shorter survival [41]. Inconsistency in the relationship between AADC expression and cancer prognosis may reflect different cancer behavior but could be attributed to study design and small patient sample size. Further 
studies are needed to clarify the role of AADC in cancer progression and prognosis.

\section{5-METHOXYTRYPTOPHAN (5-MTP) CONTROLS CANCER PROGRESSION}

5-MTP was discovered as a factor secreted by human fibroblasts for control of stress-induced cyclooxygenase-2 (COX-2) expression [42]. Purification and chemical analysis suggest that this COX-2 suppressing factor is a small molecule with an indole moiety [43]. This small molecule was named cytoguardin [43]. Comparative metabolomic analysis revealed the identity of cytoguardin as a methoxyindole derivative of Trp, i.e., 5-MTP [16]. 5-MTP inhibits cancer migration through blocking mitogen-induced expression of COX-2 and cytokines in cancer cells [16]. 5 -MTP reduces cancer growth and metastasis in vivo in a xenograft tumor model. In this murine model implanted with A549 cells, pretreatment with intraperitoneal infusion of 5-MTP reduces tumor volume and lung metastasis at 7 weeks after A549 cell implantation, accompanied by suppression of tumor COX-2 and matrix metalloprotein-9 expression [16]. 5MTP inhibits phorbol 12-myristate 13-acetate (PMA)induced COX-2 transcription in human fibroblasts by blocking binding of several transactivators including NF-kB, C/EBP 3, CREB-2 and AP-1 to the proximal region of COX-2 promoter [44]. 5-MTP inhibits cancer cell COX-2 and MMP-9 expressions at the transcriptional level.

Human fibroblasts co-incubated with A549 cells attenuate epithelial mesenchymal transition (EMT) of A549 cells and cancer cell migration [45]. Fibroblasts inhibit cancer cell EMT through 5-MTP release which inhibits EMT at least in part by blocking p38 MAPK signaling pathway [45]. This is in keeping with the action of 5-MTP on other cells: 5-MTP inhibits stressinduced macrophage activation, endothelial inflammation and smooth muscle cell proliferation through blocking p38 MAPK $[18,46,47]$. Naïve fibroblasts confer resistance to cancer development and growth [48]. However, cancer-associated fibroblasts (CAF) lose the cancer-fighting ability and are educated by cancer to become an accomplice of cancer growth [49]. The cancer promoting activity of CAFs may be attributed in part to its loss of 5-MTP production [49]. Endothelial cells (EC) are a major source of 5-MTP production. Cancer cells may convert ECs into cancer-promoting by suppressing 5-MTP production. Through 5-MTP suppression, the tumor microenvironment is proinflammatory and cancersupporting.

\section{HIOMT TRANSFECTION SWITCHES METABOLITES FROM SEROTONIN TO 5-MTP IN CANCER CELLS}

Analysis of tryptophan metabolites in conditioned medium of A549 cells indicates that serotonin and Kyn are the predominant metabolites [17]. Thus, A549 cancer cells use these two tumor-promoting Trp metabolites to advance their growth and metastasis. A549 cells express abundant TPH-1 and AADC consistent with their serotonin-producing capabilities [17]. By contrast, they express very low HIOMT, although cloning and sequence analysis of HIOMT in cancer cells provides evidence for the expression of authentic HIOMT298 isoform [17]. Transfection of A549 cells with HIOMT298 restores expression of HIOMT and production of 5-MTP. Importantly, HIOMT298 transfection is accompanied by suppression of AADC expression and reduction of serotonin production and secretion [17] (Figure 2).

HIOMT was initially identified in pineal cells. As it catalyzes conversion of $\mathrm{N}$-acetylserotonin to $\mathrm{N}$-acetyl5-methoxytryptamine (melatonin), this enzyme was named ASMT ( $\mathrm{N}$-acetylserotonin methyltransferase). ASMT is encoded by a single gene [50]. Three mRNA isoforms were detected in pineal and retinal cells as a result of alternative splicing [50,51]. The full-length mRNA codes for a 373 aa protein while exon 6-and exon 6 and 7-spliced mRNAs code for a 345 aa and 298 aa protein, respectively. Structure-function analysis shows that HIOMT345 is the active isoform which catalyzes melatonin synthesis [52]. ASMT298 is considered to be a truncated form and inactive in melatonin generation due to obliteration of binding site for the methyl donor S-adenosylmethionine (SAM) [52]. Cloning and sequence analysis confirmed that fibroblasts express HIOMT298 with sequence identical to ASMT298. It is surprising that HIOMT298, expressed in fibroblasts and vascular ECs, is active in catalyzing 5-MTP production. How this truncated enzyme catalyzes methylation of 5-HTP is a subject of interests which is being investigated.

Suppression of AADC expression by HIOMT298 transfection leads to alteration of cancer cell behavior, making it less malignant. Subcutaneous implantation of HIOMT298-transfected A549 cells in the xenograft murine tumor model results in smaller tumor growth and fewer lung metastatic nodules than implantation of control A549 cells [17]. Metabolic switch of A549 cells by HIOMT gene transfer uncovers a reciprocal relationship between serotonin and 5-MTP synthesis in 
A549 cancer cells

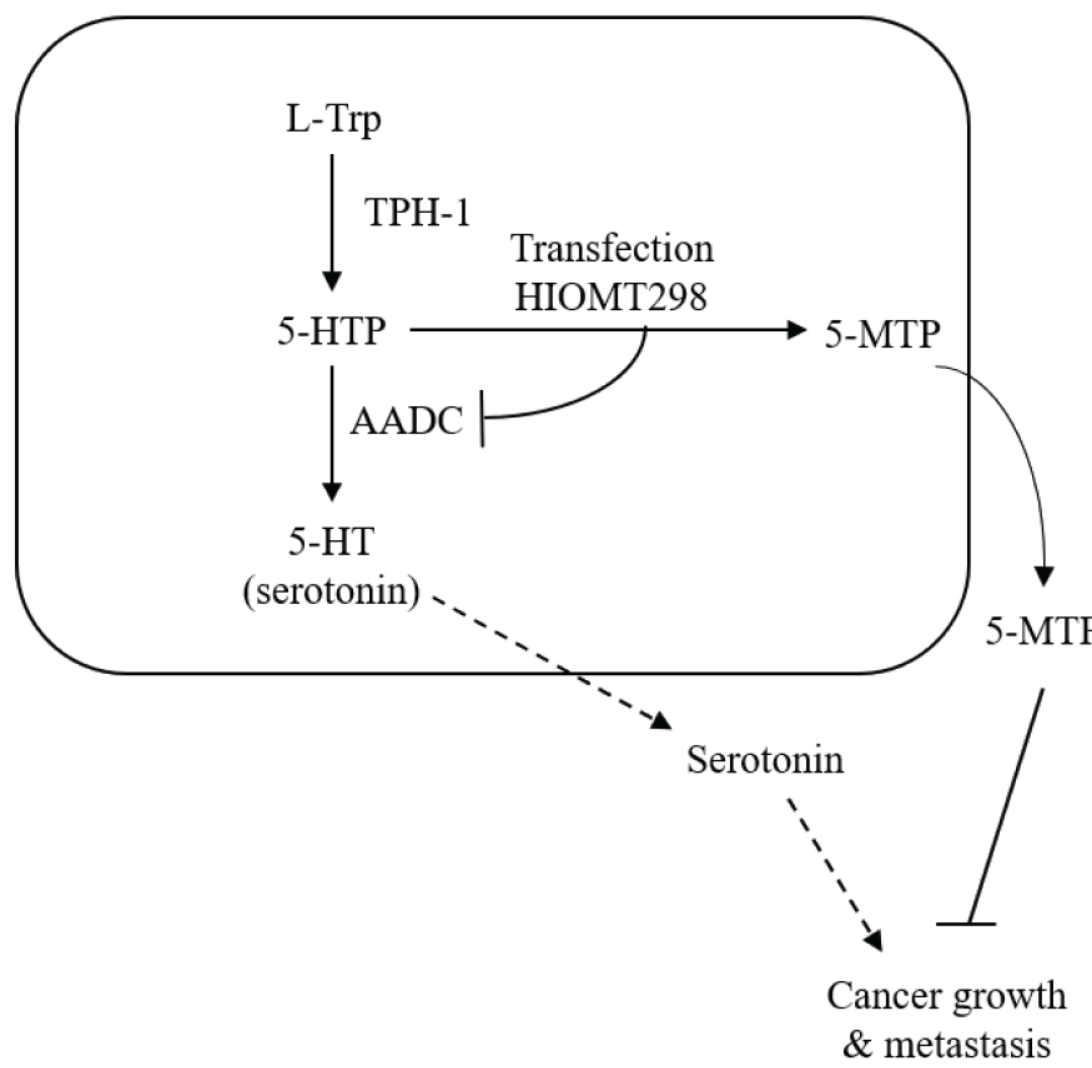

Figure 2: Metabolic switch by HIOMT transfection in A549 cancer cells. Untransfected A549 cells produce pro-tumor serotonin via AADC. HIOMT298 transfection results in suppression of AADC and shifts the metabolic pathway toward 5-MTP production.

cancer. Cancer cells reprogram TPH metabolic pathway to favor serotonin production by promoting AADC expression. Forced overexpression of HIOMT switches the metabolic program to 5-MTP production by suppressing AADC expression. The mechanism by which HIOMT298 transfection silences AADC expression is unclear and remains to be elucidated.

\section{MELATONIN INHIBITS CANCER GROWTH AND METASTASIS}

Melatonin has been extensively studied as an antitumor pharmacological agent. At pharmacological concentrations, it inhibits cell proliferation and migration of diverse cancers including breast, prostate, cervical, renal, liver, gastric, pancreatic, colorectal, pulmonary and oral cancers as well as melanoma, sarcomas, and lymphomas [53]. Results from clinical trials suggest that melatonin is useful in enhancing the efficacy of chemotherapy and improving quality of life [53]. Myriad mechanisms have been proposed to explain the anticancer effects of melatonin. They include 1). inhibition of cancer cell telomerase, and DNA damage repair, 2). control of reactive oxygen species generation, 3). blocking induced expression of cyclooxygenase-2 (COX-2), matrix metalloproteinases (MMPs) and proinflammatory cytokines, and 4). induction of cancer cell apoptosis [54]. Reported data suggest that the diverse mechanisms proposed for various cancers are probably the manifestations of a fundamental mechanism yet to be elucidated $[55,56]$.

Melatonin and 5-MTP share several common antitumor characteristics. They are highly effective in scavenging ROS and hence attenuates ROS-induced DNA damage and cell transformation [57-59]. 5-MTP was reported to upregulate manganese superoxide dismutase (MnSOD, SOD-2) and catalase [59]. Another common action is suppression of COX-2 expression. 5-MTP suppresses mitogen-cytokine- and LPS-induced COX-2 expression through inhibition of COX-2-promoting transactivators notably NF-kB $[18,46]$. Melatonin suppresses COX-2 expression in a manner similar to 5-MTP [60]. In addition, both metabolites inhibit activation of p300 histone acetyltransferase (HAT) $[18,44,60] . \quad$ p300 HAT 
acetylates histone to loosen chromatin structure and facilitate transactivator binding and acetylates transactivators such as NF-KB and C/EBP 3 to strengthen their binding to promoters resulting in enhanced transcription of COX-2 and pro-inflammatory genes [61,62]. Both metabolites block p300 HAT activity thereby suppressing pro-inflammatory gene expression. Both 5-MTP and melatonin act against a common signaling pathway: p38 MAPK. 5-MTP was reported to inhibit A549 EMT through blocking p38 MAPK [45]. Analogous to 5-MTP, melatonin inhibits breasts cancer cell invasion by repressing p38 MAPK phosphorylation [63]. Melatonin inhibits p38 MAPK activation through interaction with its membrane receptors, MT1 and MT2 which transmits signals to block p38 MAPK. 5-MTP may act through a 5-MTP receptor [18]. However, 5-MTP receptors have not been isolated and its identity remains unknown. Judging from structural similarities between 5-MTP and melatonin, it is possible that 5-MTP may act through receptors closely related to MT1 and/or MT2.

\subsection{AANAT-Derived Melatonin Defends against Cholangiocarcinoma Growth}

Despite multiple pharmacological studies showing the anticancer effect of melatonin, there are limited reports on the physiological role of 5-MTP in defending against cancer development. Melatonin is produced in

A. cholangiocytes

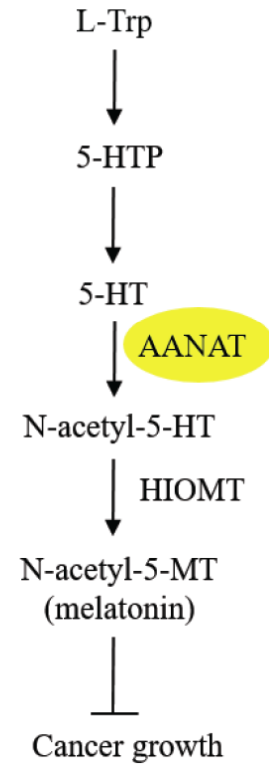

cells of intestines, liver and bile duct [64,65] but its functions in extra pineal tissues are poorly understood. A report about melatonin synthesis in cholangiocytes shed lights on the role of melatonin in defending against cancer. A key enzymatic step in melatonin synthesis is AANAT which catalyzes melatonin production in the darkness [14] (Figure 3A). The level of AANAT expression in cholangiocytes was reported to regulate melatonin synthesis [65]. AANAT expression is reduced in cholangiocarcinoma (CCA) which is accompanied by reduced melatonin synthesis and cancer progression [66] (Figure 3B). Supplement of melatonin to cultured cholangiocarcinoma cells inhibits cancer cell proliferation [66]. Furthermore, administration of melatonin to mice decreases xenograft CCA tumor volume [66]. Overexpression of AANAT in CCA cells by transfection restores melatonin synthesis and suppresses cancer cell proliferation. Melatonin may thus play an important role in defending against CCA growth.

\section{TRYPTOPHAN CATABOLIC ENZYMES AS PROGNOSTIC BIOMARKERS OF CANCER}

Several reports indicate that IDO-1 expression is increased in human cancer tissues and patient follow up studies reveal that IDO-1 expression is associated with patient outcome $[67,68]$. In a prospective study of hepatocellular cancer patients, tissue IDO expression

B. Cholangiocarcinoma (CCA) cells 
is negatively associated with patient survival: a high IDO expression is an independent prognostic factor for poor overall survival [69]. Tumor TDO overexpression was reported to be associated with poor survival in patients with breast cancer [70] and hepatocellular cancer [71]. Cancer tissue IDO-1 and TDO levels are considered to be useful cancer prognostic biomarkers.

Kyn is further catabolized to a series of bioactive metabolites with pro- or anti-cancer activities. Correlation of the catabolic enzymes with cancer progression has not been extensively investigated. One of the enzymes i.e., kynurenine 3-monooxygense (KMO) was reported to promote cancer progression and is associated with cancer prognosis [72,73]. KMO occupies a pivotal position in the main cascade of Kyn catabolism (Figure 1A). It catalyzes conversion of Kyn to 3-hydroxykynurenine (3-HK) which is converted to 3HAA by kynurenase. In a cohort study of hepatocellular cancer (HCC) patients, cancer tissue KMO expression was reported to be increased and high KMO levels were correlated with poor survival [73]. KMO overexpression in cultured HCC cells increases cancer cell proliferation, migration and invasion [73].

Prospective analysis of Kyn catabolite profile has identified 3-HAA as a prognostic factor of pancreatic carcinoma and non-small all lung cancer [74,75]. 3HAA is a potent immunosuppressive metabolite. It induces effector $T$ cell apoptosis and regulatory $T$ cell differentiation [76]. It is possible that $\mathrm{KMO}$ and its downstream catabolite 3-HAA may play an important role in cancer progression. It is worth noting that KMO promotes breast cancer progression in an enzymeindependent fashion; KMO prevents $\beta$-catenin degradation by binding to $\beta$-catenin and enhances $\beta$ catenin-mediated gene expression [72]. The reported correlation between Trp catabolic enzyme and cancer prognosis represents the tip of an iceberg. Further studies will uncover additional enzymatic and metabolite associations and provide new insights into the value of Trp metabolism as biomarkers of cancer.

\section{CONCLUSION}

A variety of cancer cells express enzymes for producing tryptophan metabolites which promote tumor growth and cancer metastasis. Two molecules, kyn produced via IDO/TDO pathway and serotonin produced via TPH-1 and AADC are of particular importance. Kyn promotes cancer cell growth through interaction with AHR expressed on cancer cells. It induces immunosuppression by reducing effector $\mathrm{T}$ cells and increasing regulatory $\mathrm{T}$ cells through interaction with AHR. Serotonin promotes cancer growth by inducing angiogenesis and cancer cell growth through interaction with serotonin-specific receptors, notably type 1 and type 2 receptors.

5-MTP is an anti-cancer metabolite produced in fibroblasts and vascular ECs. Its synthesis is catalyzed by $\mathrm{TPH}-1$ and HIOMT298. Cancer cells produce very low amounts of 5-MTP due to defective expression of HIOMT298. Co-incubation of A549 cancer cells with fibroblasts results in reduced cancer cell proliferation, migration and EMT. Administration of 5-MTP suppresses tumor growth and cancer metastasis in murine xenograft models. Transfection of A549 cancer cells with HIOMT298 restores 5-MTP production accompanied by reduction of cancer cell migration. Implantation of HIOMT-transfected A549 cells in the subcutaneous tissues of xenograft mouse model confirms reduced tumor growth and lung metastatic nodules. HIOMT298 transfection changes cancer cell tryptophan metabolic profile: switching from cancer promoting serotonin to tumor suppressing 5-MTP. 5MTP suppresses cancer growth and migration through blocking p38 MAPK, NF-kB and p300 HAT activation

Melatonin has been extensively investigated as a pharmacological agent against cancer growth. It inhibits cancer cell proliferation and migration in vitro and reduces tumor size in xenograft mouse models. Its mode of actions is analogous to 5-MTP suggesting a shared mechanism. Melatonin plays a physiological role in defending against cholangiocarcinoma. Cholangiocytes express AANAT and produce melatonin. AANAT expression is reduced in cholangiocarcinoma cells which results in production of low levels of melatonin. AANAT transfection restores cancer cell melatonin production accompanied by reduced cancer malignancy.

IDO-1 and TDO are used as therapeutic targets of cancer. Inhibitors are developed and evaluated for their anti-cancer effects. Furthermore, IDO-1 and TDO are proposed to be prognostic biomarker of cancer. On the other hand, melatonin and 5-MTP have the potentials to serve as lead compounds to develop new drugs for chemoprevention and therapy of cancer.

\section{ACKNOWLEDGEMENTS}

This work was funded by Excellent Team Research Program of Ministry of Science and Technology, Taiwan ((102-106)-2321-B-039-001; 107-2321-B-400011; 108-2321-B-400-008). 


\section{CONFLICTS OF INTERESTS}

The author declares no conflict of interest.

\section{ABBREVIATIONS}

$\operatorname{Trp}$

IDO

TDO

Kyn

$\mathrm{KMO}$

5-MTP

$\mathrm{TPH}$

AADC

DDC

AANAT

HIOMT

ASMT

SAM

5-HTP

3-HAA

AHR

ARNT

cox-2

EMT

MMP-9

MAPK

CAF

EC

ROS

CCA

$\mathrm{KMO}$

$\mathrm{HCC}$
$=$ tryptophan

$=$ indole 2, 3-dioxygenase

$=$ tryptophan 2, 3 dioxygenase

$=$ kynurenine

= kynurenine monooxygenase

= 5-methoxytryptophan

= tryptophan hydroxylase

= aromatic L-amino acid decarboxylase

= L-dopa decarboxylase

$=$ arylalkylamine $\mathrm{N}$-acetyltransferase

= hydroxyindole $\mathrm{O}$-methyltransferase

$=$ acetylserotonin methyltransferase

= S-adenosylmethionine

= 5-hydroxytryptophan

= 3-hydroxyanthranilic acid

$=$ aryl hydrocarbon receptor

$=\mathrm{AHR}$ nuclear translocator

= cyclooxygenase- 2

$=$ epithelial mesenchymal transition

$=$ matrix metalloproteinase -9

$=$ mitogen-activated protein kinase

= cancer-associated fibroblasts

$=$ endothelial cell

$=$ reactive oxygen species

$=$ cholangiocarcinoma

$=$ kynurenine monooxygenase

$=$ hepatocellular cancer
HAT

= histone acetyltransferase

Kynase

= kynurenase

QPRT

= quinolinate phosphoribosyltransferase

5-HT

= 5-hydroxytryptamine

$\mathrm{N}$-acetyl-5-MT = N-acetyl-5-methoxytryptamine

\section{REFERENCES}

[1] Munn DH, Shafizadeh E, Attwood JT, Bondarev I, Pashine A Mellor AL. Inhibition of T cell proliferation by macrophage tryptophan catabolism. J Exp Med 1999; 189(9): 1363-72. https://doi.org/10.1084/jem.189.9.1363

[2] Terness P, Bauer TM, Röse L, Dufter C, Watzlik A, Simon H, et al. Inhibition of allogeneic $\mathrm{T}$ cell proliferation by indoleamine 2,3-dioxygenase-expressing dendritic cells: mediation of suppression by tryptophan metabolites. J Exp Med 2002; 196(4): 447-57. https://doi.org/10.1084/jem.20020052

[3] Munn DH, Zhou M, Attwood JT, Bondarev I, Conway SJ, Marshall $\mathrm{B}$, et al. Prevention of allogeneic fetal rejection by tryptophan catabolism. Science 1998; 281(5380): 1191-3. https://doi.org/10.1126/science.281.5380.1191

[4] Schmidt SK, Müller A, Heseler K, Woite C, Spekker K MacKenzie CR, et al. Antimicrobial and immunoregulatory properties of human tryptophan 2,3-dioxygenase. Eur J Immunol 2009; 39(10): 2755-64. https://doi.org/10.1002/eji.200939535

[5] Chon SY, Hassanain HH, Gupta SL. Cooperative role of interferon regulatory factor 1 and p91 (STAT1) response elements in interferon-gamma-inducible expression of human indoleamine 2,3-dioxygenase gene. J Biol Chem 1996; 271(29): 17247-52. https://doi.org/10.1074/jbc.271.29.17247

[6] Kanai M, Funakoshi H, Takahashi H, Hayakawa T, Mizuno S, Matsumoto $\mathrm{K}$, et al. Tryptophan 2,3-dioxygenase is a key modulator of physiological neurogenesis and anxiety-related behavior in mice. Mol Brain 2009; 2:8. https://doi.org/10.1186/1756-6606-2-8

[7] Cervenka I, Agudelo LZ, Ruas JL. Kynurenines: Tryptophan's metabolites in exercise, inflammation, and mental health. Science 2017; 357(6349): eaaf9794 https://doi.org/10.1126/science.aaf9794

[8] Lovenberg W, Jequier E, Sjoerdsma A. Tryptophan hydroxylation: measurement in pineal gland, brainstem, and carcinoid tumor. Science 1967; 155(3759): 217-9. https://doi.org/10.1126/science.155.3759.217

[9] Walther DJ, Peter JU, Bashammakh S, Hörtnagl H, Voits M, Fink $\mathrm{H}$, et al. Synthesis of serotonin by a second tryptophan hydroxylase isoform. Science 2003; 299(5603): 76. https://doi.org/10.1126/science.1078197

[10] Christenson JG, Dairman W, Udenfriend S. On the identity of DOPA decarboxylase and 5-hydroxytryptophan decarboxylase (immunological titration-aromatic L-amino acid decarboxylase-serotonin-dopamine-norepinephrine). Proc Natl Acad Sci U S A 1972; 69(2): 343-7.

https://doi.org/10.1073/pnas.69.2.343

[11] Berger M, Gray JA, Roth BL. The expanded biology of serotonin. Annu Rev Med 2009; 60: 355-66. https://doi.org/10.1146/annurev.med.60.042307.110802

El-Merahbi R, Löffler M, Mayer A, Sumara G. The roles of peripheral serotonin in metabolic homeostasis. FEBS Lett 2015; 589(15): 1728-34.

https://doi.org/10.1016/j.febslet.2015.05.054 
[13] Axelrod J, Weissbach $\mathrm{H}$. Enzymatic O-methylation of $\mathrm{N}$ acetylserotonin to melatonin. Science 1960; 131(3409): 1312. https://doi.org/10.1126/science.131.3409.1312

[14] Klein DC. Arylalkylamine N-acetyltransferase: "the Timezyme". J Biol Chem 2007; 282(7): 4233-7. https://doi.org/10.1074/jbc.R600036200

[15] Hardeland R, Pandi-Perumal SR, Cardinali DP. Melatonin. Int J Biochem Cell Biol 2006; 38(3): 313-6. https://doi.org/10.1016/j.biocel.2005.08.020

[16] Cheng HH, Kuo CC, Yan JL, Chen HL, Lin WC, Wang KH, et al. Control of cyclooxygenase-2 expression and tumorigenesis by endogenous 5-methoxytryptophan. Proc Natl Acad Sci U S A 2012; 109(33): 13231-6. https://doi.org/10.1073/pnas.1209919109

[17] Chen HL, Yuan CY, Cheng HH, Chang TC, Huang SK, Kuo $\mathrm{CC}$, et al. Restoration of hydroxyindole O-methyltransferase levels in human cancer cells induces a tryptophan-metabolic switch and attenuates cancer progression. J Biol Chem 2018; 293(28): 11131-11142. https://doi.org/10.1074/jbc.RA117.000597

[18] Wang YF, Hsu YJ, Wu HF, Lee GL, Yang YS, Wu JY, et al. Endothelium-Derived 5-Methoxytryptophan Is a Circulating Anti-Inflammatory Molecule That Blocks Systemic Inflammation. Circ Res 2016; 119(2): 222-36. https://doi.org/10.1161/CIRCRESAHA.116.308559

[19] Théate I, van Baren N, Pilotte L, Moulin P, Larrieu P, Renauld JC, et al. Extensive profiling of the expression of the indoleamine 2,3-dioxygenase 1 protein in normal and tumoral human tissues. Cancer Immunol Res 2015; 3(2): 161-72. https://doi.org/10.1158/2326-6066.CIR-14-0137

[20] Abdel-Magid AF. Targeting the Inhibition of Tryptophan 2,3Dioxygenase (TDO-2) for Cancer Treatment. ACS Med Chem Lett 2016; 8(1): 11-13. https://doi.org/10.1021/acsmedchemlett.6b00458

[21] Du L, Xing Z, Tao B, Li T, Yang D, Li W, et al. Both IDO1 and TDO contribute to the malignancy of gliomas via the KynAhR-AQP4 signaling pathway. Signal Transduct Target Ther 2020; 5(1): 10.

https://doi.org/10.1038/s41392-019-0103-4

[22] Opitz CA, Litzenburger UM, Sahm F, Ott M, Tritschler I, Trump S, et al. An endogenous tumour-promoting ligand of the human aryl hydrocarbon receptor. Nature 2011; 478(7368): 197-203.

https://doi.org/10.1038/nature10491

[23] Murray IA, Patterson AD, Perdew GH. Aryl hydrocarbon receptor ligands in cancer: friend and foe. Nat Rev Cancer 2014; 14(12): 801-14 https://doi.org/10.1038/nrc3846

[24] Munn DH, Mellor AL. IDO in the Tumor Microenvironment: Inflammation, Counter-Regulation, and Tolerance. Trends Immunol 2016; 37(3): 193-207.

https://doi.org/10.1016/j.it.2016.01.002

[25] Frumento G, Rotondo R, Tonetti M, Damonte G, Benatti U, Ferrara GB. Tryptophan-derived catabolites are responsible for inhibition of $\mathrm{T}$ and natural killer cell proliferation induced by indoleamine 2,3-dioxygenase. J Exp Med 2002; 196(4): 459-68.

https://doi.org/10.1084/jem.20020121

[26] Fallarino F, Grohmann U, Vacca C, Bianchi R, Orabona C, Spreca A, et al. T cell apoptosis by tryptophan catabolism. Cell Death Differ 2002; 9(10): 1069-77. https://doi.org/10.1038/sj.cdd.4401073

[27] Orabona C, Puccetti P, Vacca C, Bicciato S, Luchini A, Fallarino $\mathrm{F}$, et al. Toward the identification of a tolerogenic signature in IDO-competent dendritic cells. Blood 2006; 107(7): 2846-54

https://doi.org/10.1182/blood-2005-10-4077
[28] Hill M, Tanguy-Royer S, Royer P, Chauveau C, Asghar K, Tesson L, et al. IDO expands human CD4+CD25high regulatory $\mathrm{T}$ cells by promoting maturation of LPS-treated dendritic cells. Eur J Immunol 2007; 37(11): 3054-62.

https://doi.org/10.1002/eji.200636704

[29] Xue P, Fu J, Zhou Y. The Aryl Hydrocarbon Receptor and Tumor Immunity. Front Immunol 2018; 9: 286. https://doi.org/10.3389/fimmu.2018.00286

[30] Nocito A, Dahm F, Jochum W, Jang JH, Georgiev P, Bader $\mathrm{M}$, et al. Serotonin regulates macrophage-mediated angiogenesis in a mouse model of colon cancer allografts. Cancer Res 2008; 68(13): 5152-8. https://doi.org/10.1158/0008-5472.CAN-08-0202

[31] Alpini G, Invernizzi P, Gaudio E, Venter J, Kopriva S, Bernuzzi $F$, et al. Serotonin metabolism is dysregulated in cholangiocarcinoma, which has implications for tumor growth. Cancer Res 2008; 68(22): 9184-93. https://doi.org/10.1158/0008-5472.CAN-08-2133

[32] Soll C, Jang JH, Riener MO, Moritz W, Wild PJ, Graf R, et al Serotonin promotes tumor growth in human hepatocellular cancer. Hepatology 2010; 51(4): 1244-54.

https://doi.org/10.1002/hep.23441

[33] Leoncikas $\mathrm{V}$, Wu $\mathrm{H}$, Ward LT, Kierzek AM, Plant NJ. Generation of 2,000 breast cancer metabolic landscapes reveals a poor prognosis group with active serotonin production. Sci Rep 2016; 6: 19771. https://doi.org/10.1038/srep19771

[34] Sarrouilhe D, Clarhaut J, Defamie N, Mesnil M. Serotonin and cancer: what is the link? Curr Mol Med 2015; 15(1): 6277.

https://doi.org/10.2174/1566524015666150114113411

[35] Ichinose H, Sumi-Ichinose C, Ohye T, Hagino Y, Fujita K, Nagatsu T. Tissue-specific alternative splicing of the first exon generates two types of mRNAs in human aromatic Lamino acid decarboxylase. Biochemistry 1992; 31(46): 11546-50.

https://doi.org/10.1021/bi00161a036

[36] Albert VR, Lee MR, Bolden AH, Wurzburger RJ, Aguanno A Distinct promoters direct neuronal and nonneuronal expression of rat aromatic L-amino acid decarboxylase. Proc Natl Acad Sci U S A 1992; 89(24): 12053-7. https://doi.org/10.1073/pnas.89.24.12053

[37] O'Malley KL, Harmon S, Moffat M, Uhland-Smith A, Wong S The human aromatic L-amino acid decarboxylase gene can be alternatively spliced to generate unique protein isoforms. J Neurochem 1995; 65(6): 2409-16. https://doi.org/10.1046/j.1471-4159.1995.65062409.x

[38] Vachtenheim J, Novotná H. Expression of the aromatic Lamino acid decarboxylase mRNA in human tumour cell lines of neuroendocrine and neuroectodermal origin. Eur $\mathrm{J}$ Cancer 1997; 33(14): 2411-7. https://doi.org/10.1016/S0959-8049(97)00302-X

[39] Bepler G, Jaques G, Koehler A, Gropp C, Havemann K. Markers and characteristics of human SCLC cell lines. Neuroendocrine markers, classical tumor markers, and chromosomal characteristics of permanent human small cell lung cancer cell lines. J Cancer Res Clin Oncol 1987; 113(3): 253-9. https://doi.org/10.1007/BF00396382

[40] Kontos CK, Papadopoulos IN, Fragoulis EG, Scorilas A Quantitative expression analysis and prognostic significance of L-DOPA decarboxylase in colorectal adenocarcinoma. Br J Cancer 2010; 102(9): 1384-90. https://doi.org/10.1038/sj.bjc.6605654

[41] Koutalellis G, Stravodimos K, Avgeris M, Mavridis K, Scorilas A, Lazaris A, et al. L-dopa decarboxylase (DDC) gene expression is related to outcome in patients with prostate cancer. BJU Int 2012; 110(6 Pt B): E267-73. https://doi.org/10.1111/j.1464-410X.2012.11152.x 
[42] Gilroy DW, Saunders MA, Sansores-Garcia L, MatijevicAleksic N, Wu KK. Cell cycle-dependent expression of cyclooxygenase-2 in human fibroblasts. FASEB J 2001; 15(2): 288-90.

\section{https://doi.org/10.1096/fj.00-0573fje}

[43] Deng WG, Saunders M, Gilroy D, He XZ, Yeh H, Zhu Y, et al. Purification and characterization of a cyclooxygenase-2 and angiogenesis suppressing factor produced by human fibroblasts. FASEB J 2002; 16(10): 1286-8.

https://doi.org/10.1096/fj.01-0844fje

[44] Cheng HH, Wang KH, Chu LY, Chang TC, Kuo CC, Wu KK. Quiescent and proliferative fibroblasts exhibit differential p300 HAT activation through control of 5-methoxytryptophan production. PLoS One 2014; 9(2): e88507. https://doi.org/10.1371/journal.pone.0088507

[45] Cheng HH, Chu LY, Chiang LY, Chen HL, Kuo CC, Wu KK. Inhibition of cancer cell epithelial mesenchymal transition by normal fibroblasts via production of 5-methoxytryptophan. Oncotarget 2016; 7(21): 31243-56 https://doi.org/10.18632/oncotarget.9111

[46] Chu LY, Wang YF, Cheng HH, Kuo CC, Wu KK. Endothelium-Derived 5-Methoxytryptophan Protects Endothelial Barrier Function by Blocking p38 MAPK Activation. PLoS One 2016; 11(3): e0152166. https://doi.org/10.1371/journal.pone.0152166

[47] Ho YC, Wu ML, Su CH, Chen $\mathrm{CH}, \mathrm{Ho} \mathrm{HH}$, Lee GL, et al. A Novel Protective Function of 5-Methoxytryptophan in Vascular Injury. Sci Rep 2016; 6: 25374.

https://doi.org/10.1038/srep25374

[48] Flaberg E, Markasz L, Petranyi G, Stuber G, Dicso F, Alchihabi $\mathrm{N}$, et al. High-throughput live-cell imaging reveals differential inhibition of tumor cell proliferation by human fibroblasts. Int J Cancer 2011; 128(12): 2793-802. https://doi.org/10.1002/ijc.25612

[49] Olumi AF, Grossfeld GD, Hayward SW, Carroll PR, Tlsty TD, Cunha GR. Carcinoma-associated fibroblasts direct tumor progression of initiated human prostatic epithelium. Cancer Res 1999; 59(19): 5002-11.

[50] Rodriguez IR, Mazuruk K, Schoen TJ, Chader GJ. Structural analysis of the human hydroxyindole-O-methyltransferase gene. Presence of two distinct promoters. J Biol Chem 1994; 269(50): 31969-77. https://doi.org/10.1016/S0021-9258(18)31790-3

[51] Donohue SJ, Roseboom PH, Illnerova H, Weller JL, Klein DC. Human hydroxyindole-O-methyltransferase: presence of LINE-1 fragment in a cDNA clone and pineal mRNA. DNA Cell Biol 1993; 12(8): 715-27. https://doi.org/10.1089/dna.1993.12.715

[52] Botros HG, Legrand P, Pagan C, Bondet V, Weber P, BenAbdallah $\mathrm{M}$, et al. Crystal structure and functional mapping of human ASMT, the last enzyme of the melatonin synthesis pathway. J Pineal Res 2013; 54(1): 46-57. https://doi.org/10.1111/j.1600-079X.2012.01020.x

[53] Li Y, Li S, Zhou Y, Meng X, Zhang JJ, Xu DP, et al. Melatonin for the prevention and treatment of cancer. Oncotarget 2017; 8(24): 39896-39921. https://doi.org/10.18632/oncotarget.16379

[54] Reiter RJ. Mechanisms of cancer inhibition by melatonin. J Pineal Res 2004; 37(3): 213-4 https://doi.org/10.1111/j.1600-079X.2004.00165.x

[55] Reiter RJ, Rosales-Corral SA, Tan DX, Acuna-Castroviejo D, Qin L, Yang SF, et al. Melatonin, a Full Service Anti-Cancer Agent: Inhibition of Initiation, Progression and Metastasis. Int J Mol Sci 2017; 18(4): 843. https://doi.org/10.3390/ijms18040843

[56] Hill SM, Belancio VP, Dauchy RT, Xiang S, Brimer S, Mao L, et al. Melatonin: an inhibitor of breast cancer. Endocr Relat Cancer 2015; 22(3): R183-204. https://doi.org/10.1530/ERC-15-0030
[57] Martín V, Herrera F, Carrera-Gonzalez P, García-Santos G, Antolín I, Rodriguez-Blanco J, et al. Intracellular signaling pathways involved in the cell growth inhibition of glioma cells by melatonin. Cancer Res 2006; 66(2): 1081-8.

\section{https://doi.org/10.1158/0008-5472.CAN-05-2354}

[58] Galano A, Reiter RJ. Melatonin and its metabolites vs oxidative stress: From individual actions to collective protection. J Pineal Res 2018; 65(1): e12514. https://doi.org/10.1111/jpi.12514

[59] Chang TC, Hsu MF, Shih CY, Wu KK. 5-methoxytryptophan protects MSCs from stress induced premature senescence by upregulating FoxO3a and mTOR. Sci Rep 2017; 7(1): 11133. https://doi.org/10.1038/s41598-017-11077-4

[60] Wang J, Xiao X, Zhang Y, Shi D, Chen W, Fu L, et al. Simultaneous modulation of COX-2, p300, Akt, and Apaf-1 signaling by melatonin to inhibit proliferation and induce apoptosis in breast cancer cells. J Pineal Res 2012; 53(1): 77-90. https://doi.org/10.1111/j.1600-079X.2012.00973.x

[61] Deng WG, Zhu Y, Wu KK. Up-regulation of p300 binding and p50 acetylation in tumor necrosis factor-alpha-induced cyclooxygenase-2 promoter activation. J Biol Chem 2003; 278(7): 4770-7. https://doi.org/10.1074/jbc.M209286200

[62] Deng WG, Zhu Y, Wu KK. Role of p300 and PCAF in regulating cyclooxygenase-2 promoter activation by inflammatory mediators. Blood 2004; 103(6): 2135-42. https://doi.org/10.1182/blood-2003-09-3131

[63] Mao L, Yuan L, Slakey LM, Jones FE, Burow ME, Hill SM. Inhibition of breast cancer cell invasion by melatonin is mediated through regulation of the p38 mitogen-activated protein kinase signaling pathway. Breast Cancer Res 2010; 12(6): R107.

https://doi.org/10.1186/bcr2794

[64] Huether G. The contribution of extrapineal sites of melatonin synthesis to circulating melatonin levels in higher vertebrates. Experientia 1993; 49(8): 665-70. https://doi.org/10.1007/BF01923948

[65] Renzi A, DeMorrow S, Onori P, Carpino G, Mancinelli R, Meng $\mathrm{F}$, et al. Modulation of the biliary expression of arylalkylamine $\mathrm{N}$-acetyltransferase alters the autocrine proliferative responses of cholangiocytes in rats. Hepatology 2013; 57(3): 1130-41. https://doi.org/10.1002/hep.26105

[66] Han Y, Demorrow S, Invernizzi P, Jing Q, Glaser S, Renzi A et al. Melatonin exerts by an autocrine loop antiproliferative effects in cholangiocarcinoma: its synthesis is reduced favoring cholangiocarcinoma growth. Am J Physiol Gastrointest Liver Physiol 2011; 301(4): G623-33. https://doi.org/10.1152/ajpgi.00118.2011

[67] Pan K, Wang H, Chen MS, Zhang HK, Weng DS, Zhou J, et al. Expression and prognosis role of indoleamine 2,3dioxygenase in hepatocellular carcinoma. J Cancer Res Clin Oncol 2008; 134(11): 1247-53.

https://doi.org/10.1007/s00432-008-0395-1

[68] Yu CP, Fu SF, Chen X, Ye J, Ye Y, Kong LD, et al. The Clinicopathological and Prognostic Significance of IDO1 Expression in Human Solid Tumors: Evidence from a Systematic Review and Meta-Analysis. Cell Physiol Biochem 2018; 49(1): 134-143. https://doi.org/10.1159/000492849

[69] Wang Y, Yao R, Zhang L, Xie X, Chen R, Ren Z. IDO and intra-tumoral neutrophils were independent prognostic factors for overall survival for hepatocellular carcinoma. J Clin Lab Anal 2019; 33(5): e22872. https://doi.org/10.1002/jcla.22872

[70] Liu Q, Zhai J, Kong X, Wang X, Wang Z, Fang $Y$, et al. Comprehensive Analysis of the Expression and Prognosis for 
TDO2 in Breast Cancer. Mol Ther Oncolytics 2020; 17: 153168.

https://doi.org/10.1016/j.omto.2020.03.013

[71] Li S, Li L, Wu J, Song F, Qin Z, Hou L, et al. TDO Promotes Hepatocellular Carcinoma Progression. Onco Targets Ther 2020; 13: 5845-5855.

https://doi.org/10.2147/OTT.S252929

[72] Huang TT, Tseng LM, Chen JL, Chu PY, Lee CH, Huang CT, et al. Kynurenine 3-monooxygenase upregulates pluripotent genes through $\beta$-catenin and promotes triple-negative breast cancer progression. EBioMedicine 2020; 54: 102717. https://doi.org/10.1016/j.ebiom.2020.102717

[73] Jin H, Zhang $\mathrm{Y}$, You H, Tao X, Wang C, Jin G, et al. Prognostic significance of kynurenine 3-monooxygenase and effects on proliferation, migration, and invasion of human hepatocellular carcinoma. Sci Rep 2015; 5: 10466. https://doi.org/10.1038/srep10466
[74] Huang JY, Butler LM, Midttun Ø, Ulvik A, Wang R, Jin A, et al. A prospective evaluation of serum kynurenine metabolites and risk of pancreatic cancer. PLoS One 2018; 13(5): e0196465.

https://doi.org/10.1371/journal.pone.0196465

[75] Karayama M, Masuda J, Mori K, Yasui H, Hozumi H, Suzuki $\mathrm{Y}$, et al. Comprehensive assessment of multiple tryptophan metabolites as potential biomarkers for immune checkpoint inhibitors in patients with non-small cell lung cancer. Clin Transl Oncol 2020. https://doi.org/10.1007/s12094-020-02421-8

[76] Fallarino F, Grohmann U, Vacca C, Bianchi R, Orabona C, Spreca A, et al. T cell apoptosis by tryptophan catabolism. Cell Death Differ 2002; 9(10): 1069-77. https://doi.org/10.1038/sj.cdd.4401073

Received on 29-04-2021

https://doi.org/10.30683/1927-7229.2021.10.01

(C) 2021 Kenneth K. Wu; Licensee Neoplasia Research.

This is an open access article licensed under the terms of the Creative Commons Attribution Non-Commercial License (http://creativecommons.org/licenses/by-nc/3.0/) which permits unrestricted, non-commercial use, distribution and reproduction in any medium, provided the work is properly cited. 